\title{
Predictive value of serum CTRP9 and STIM1 for restenosis after cerebrovascular stent implantation and its relationship with vasoactive substances and inflammatory cytokines
}

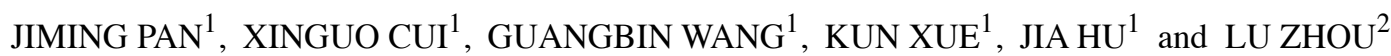 \\ ${ }^{1}$ Department of Neurosurgery, Yantaishan Hospital; ${ }^{2}$ Clinical Laboratory, \\ Yantai Hospital of Traditional Chinese Medicine, Yantai, Shandong 264000, P.R. China
}

Received October 2, 2019; Accepted July 16, 2020

DOI: $10.3892 /$ etm.2020.9104

\begin{abstract}
Predictive value of serum complement Clq tumor necrosis factor-related protein 9 (CTRP9) and serum stromal interaction molecule 1 (STIM1) was investigated for restenosis after cerebrovascular stent implantation, as well as its relationship with vasoactive substances and inflammatory cytokines. In this prospective study, 128 patients with cerebral infarction treated with cerebrovascular stent implantation in Yantaishan Hospital were recruited. A total of 66 cases with restenosis after cerebrovascular stent implantation were included in group A, and 62 cases without stenosis were included in group B. Serum CTRP9 and STIM1 levels were measured by enzyme-linked immunosorbent assay (ELISA). ROC curves of serum CTRP9 and STIM1 levels in patients with postoperative restenosis were drawn. The vasoactive substances nitric oxide (NO), tumor necrosis factor $\alpha$ (TNF- $\alpha$ ) and interleukin-6 (IL-6) were analyzed by ELISA. The correlation of serum CTRP9, STIM1 levels and NO, TNF- $\alpha$, IL- 6 were analyzed by Pearson correlation coefficient. Serum CTRP9 and NO levels in group A were significantly lower than those in group B. The levels of serum STIM1, TNF- $\alpha$ and IL-6 in group A were significantly higher than those in group $\mathrm{B}(\mathrm{P}<0.001)$. The sensitivity and specificity of serum CTRP9 level in the diagnosis of restenosis after cerebrovascular stent implantation were, respectively, 59.68 and $75.76 \%$. Those of serum STIM1 were, respectively, 87.10 and $46.97 \%$ and those of the combination of serum CTRP9 and STIM1 were 90.32 and $48.48 \%$. Serum CTRP9 level was positively correlated with $\mathrm{NO}$, and negatively correlated with TNF- $\alpha$ and IL-6. STIM1 was positively correlated with TNF- $\alpha$ and IL-6, and negatively correlated with NO (P<0.001). Serum CTRP9 level was significantly decreased in patients with restenosis after cerebrovascular stent implantation, while STIM1
\end{abstract}

Correspondence to: Dr Lu Zhou, Clinical Laboratory, Yantai Hospital of Traditional Chinese Medicine, 39 Xingfu Road, Zhifu, Yantai, Shandong 264000, P.R. China

E-mail: zlu41a@163.com

Key words: restenosis after cerebrovascular stent implantation, CTRP9, STIM1, nitric oxide, IL-6, TNF- $\alpha$ level was significantly up-regulated. Both were correlated with the change of NO, IL- 6 and TNF- $\alpha$ levels, therefore they could be used as biological indicators for prediction of restenosis after cerebrovascular stent implantation.

\section{Introduction}

With the change in people's quality of life, the incidence of cerebral infarction increases, leading to an increase of mortality in patients with cardiovascular and cerebrovascular diseases (1). For stroke patients, conventional treatment mainly includes anticoagulation, thrombolysis and other supportive treatments (2). In recent years, cerebrovascular stent implantation has been applied in the interventional therapy of cerebral infarction, which has achieved great success and has been widely applied in clinical practice (3). However, cerebrovascular restenosis is often complicated during the later operation of stent implantation, which is one of the difficulties that hinder the recovery of patients after surgery. The disease deteriorates severely, and the mortality is high $(4,5)$. Therefore, it is urgent to study predictors of restenosis after cerebrovascular stent implantation to save patients' lives and assist their recovery.

It has been found that serum complement Clq tumor necrosis factor-related protein 9 (CTRP9) is one of the adipocyte factors synthesized and released by adipose tissue. It is highly homologous with adiponectin during evolution, has the functions of regulating inflammatory response, anti-endothelial dysfunction, protecting myocardium and the metabolism of lipolysaccharide, and plays a role in regulating the stability of the body (6-10). Stromal interaction molecule 1 (STIM1) is closely related to endothelial cell function and homing of endothelial progenitor cells. The increased content may lead to injury of endothelial cells and delay of stent reendothelialization after cardiac stent implantation $(11,12)$. However, there are relatively few studies on the effect of serum CTRP9 and STIM1 on restenosis after cerebrovascular stent implantation. Therefore, this study investigated the predictive value of serum CTRP9 and STIM1 for restenosis after cerebrovascular stent implantation, and their relationship with the vasoactive substances nitric oxide (NO), tumor necrosis factor $\alpha$ (TNF- $\alpha$ ), and interleukin-6 (IL-6) to validate biological indicators of early restenosis in cerebrovascular stent implantation. 
Table I. Baseline data of patients.

\begin{tabular}{|c|c|c|c|c|}
\hline Characteristics & Group A (n=66) & Group B $(n=62)$ & $\chi^{2}$ test & P-value \\
\hline Age (years) & $53.58 \pm 7.36$ & $54.36 \pm 7.86$ & 0.580 & 0.563 \\
\hline $\operatorname{Sex}[\mathrm{n}(\%)]$ & & & 0.058 & 0.809 \\
\hline Male & $38(57.58)$ & $37(59.68)$ & & \\
\hline Female & $28(42.42)$ & $25(40.32)$ & & \\
\hline Height $(\mathrm{cm})$ & $163.53 \pm 7.45$ & $164.35 \pm 7.14$ & 0.635 & 0.527 \\
\hline $\operatorname{BMI}\left(\mathrm{kg} / \mathrm{m}^{2}\right)$ & $25.64 \pm 2.34$ & $25.57 \pm 2.28$ & 0.171 & 0.864 \\
\hline Smoking history [n (\%)] & $48(72.73)$ & $44(70.97)$ & 0.049 & 0.825 \\
\hline Drinking history [n (\%)] & $51(77.27)$ & $49(79.03)$ & 0.058 & 0.810 \\
\hline \multicolumn{5}{|l|}{ Past history [n (\%)] } \\
\hline Hypertension & $25(37.88)$ & $22(35.48)$ & 0.079 & 0.779 \\
\hline Coronary heart disease & $14(21.21)$ & $11(17.74)$ & 0.245 & 0.621 \\
\hline Diabetes mellitus & $17(25.76)$ & $15(24.19)$ & 0.042 & 0.838 \\
\hline Hyperlipidemia & $16(24.24)$ & $17(27.42)$ & 0.169 & 0.681 \\
\hline
\end{tabular}

\section{Patients and methods}

Baseline data. In this prospective study, 128 cases of cerebral infarction treated with cerebrovascular stent implantation in Yantaishan Hospital (Yantai, China) from March 2013 to January 2016 were selected as experimental subjects. A total of 66 cases with restenosis after cerebrovascular stent implantation were included in group A, while 62 cases without stenosis were included in group B. There were 75 males and 53 females, with an average age of $54.82 \pm 7.74$. There was no significant difference in gender, age, etc. between the two groups (all $\mathrm{P}>0.05$; Table I).

Inclusion and exclusion criteria. Inclusion criteria: Patients diagnosed with cerebral infarction by cerebral angiography technology.

Exclusion criteria: Patients with abnormal function of previous coagulation; patients with contraindications of stent implantation; patients complicated with endocrine system diseases; patients with severe hepatic and kidney function obstacle; patients with cognitive impairment or communication barrier; patients with poor compliance.

This study was approved by the Ethics Committee of Yantaishan Hospital (approval no. YTSH20130301). All the patients and their families were well informed, and a signed informed consent was obtained.

Experimental reagents and materials. CTRP9 kit was purchased from Aviscera Bioscience Co., (XY-RD191180200R). STIM1 kit was purchased from Shanghai Renjie Biotechnology Co., Ltd. (RJ12740). NO kit and IL-6 kit were purchased from Wuhan Mskbio Biotechnology Co., Ltd. (KT76381, KT63251). TNF- $\alpha$ kit was purchased from Nanjing Camilo Bioengineering Co., Ltd. (H-KMLJ37541).

Detection of serum CTRP9 and STIM1. Serum expression levels of CTRP9 and STIM1 of study subjects were measured by ELISA. After 1 month of cerebrovascular stent implantation, $5 \mathrm{ml}$ of patient's venous blood was collected and centrifuged at room temperature for $8 \mathrm{~min}$. The serum was separated at $1,500 \mathrm{x} \mathrm{g}$, sealed and stored at $-80^{\circ} \mathrm{C}$ for testing. A total of $100 \mu \mathrm{l}$ of serum sample was added to the orifice coated with anti-CTRP9 antibody. A blank well and a standard well were set up. The CTRP9 antibody was added at $37^{\circ} \mathrm{C}$ for reaction for $90 \mathrm{~min}$, and the liquid was discarded, then dried and washed repeatedly three times. A total of $150 \mu \mathrm{l}$ of the chromogenic reagent was added at $37^{\circ} \mathrm{C}$ for reaction for $30 \mathrm{~min}$. A total of $50 \mu \mathrm{l}$ of the mixture was taken out, mixed and put into enzyme-labelling measuring instrument to measure the average optical density of each well by $500 \mathrm{~nm}$ wavelength. STIM1, NO, TNF- $\alpha$ and IL-6 levels were detected as above in strict accordance with the ELISA kit instructions.

Observation indexes. Comparison of serum CTRP9 and STIM1 levels of patients in the two groups; comparison of NO, TNF- $\alpha$ and IL- 6 levels of patients in the two groups; comparison of diagnostic efficacy of serum CTRP9, STIM1 and their combination; analysis of the correlation between serum CTRP9, STIM1 levels and NO, TNF- $\alpha$, IL-6 levels.

Statistical methods. SPSS 19.0 statistical software (Shanghai Kabei Information Technology Co., Ltd.) was used for statistical analysis of experimental data. The counting data were tested by chi-square test. Measurement data were expressed as mean \pm standard deviation. $T$ test was used for comparison between two groups. The diagnostic value of CTRP9, STIM1 and their combination in restenosis after cerebrovascular stent implantation was analyzed by ROC. Correlation analysis was performed by Pearson's correlation coefficient. Graphpad Prism8 was used for image rendering. $\mathrm{P}<0.05$ was considered to indicate a statistically significant difference.

\section{Results}

Comparison of serum CTRP9 and STIM1 levels of patients in the two groups. Serum CTRP9 level in group A was 
Table II. Comparison of serum CTRP9 and STIM1 levels in the two groups.

\begin{tabular}{|c|c|c|c|c|}
\hline Factors & Group A $(n=66)$ & Group B $(n=62)$ & $\mathrm{t}$ & P-value \\
\hline CTRP9 (ng/ml) & $159.64 \pm 32.55$ & $184.53 \pm 34.53$ & 4.190 & $<0.001$ \\
\hline STIM1 (U/1) & $12.15 \pm 1.44$ & $11.31 \pm 0.92$ & 3.905 & $<0.001$ \\
\hline
\end{tabular}

Table III. Comparison of NO, TNF- $\alpha$, IL-6 levels of patients in the two groups.

\begin{tabular}{|c|c|c|c|c|}
\hline Factors & Group A (n=66) & Group B $(n=62)$ & $\mathrm{t}$ & P-value \\
\hline $\mathrm{NO}(\mathrm{mol} / \mathrm{l})$ & $156.57 \pm 12.58$ & $172.73 \pm 13.83$ & 6.901 & $<0.001$ \\
\hline TNF- $\alpha(n g / l)$ & $10.52 \pm 1.68$ & $7.78 \pm 0.93$ & 11.310 & $<0.001$ \\
\hline IL-6 (ng/l) & $98.75 \pm 13.53$ & $83.48 \pm 9.84$ & 7.263 & $<0.001$ \\
\hline
\end{tabular}

NO, nitric oxide; TNF- $\alpha$, tumor necrosis factor $\alpha$; IL-6; interleukin-6.
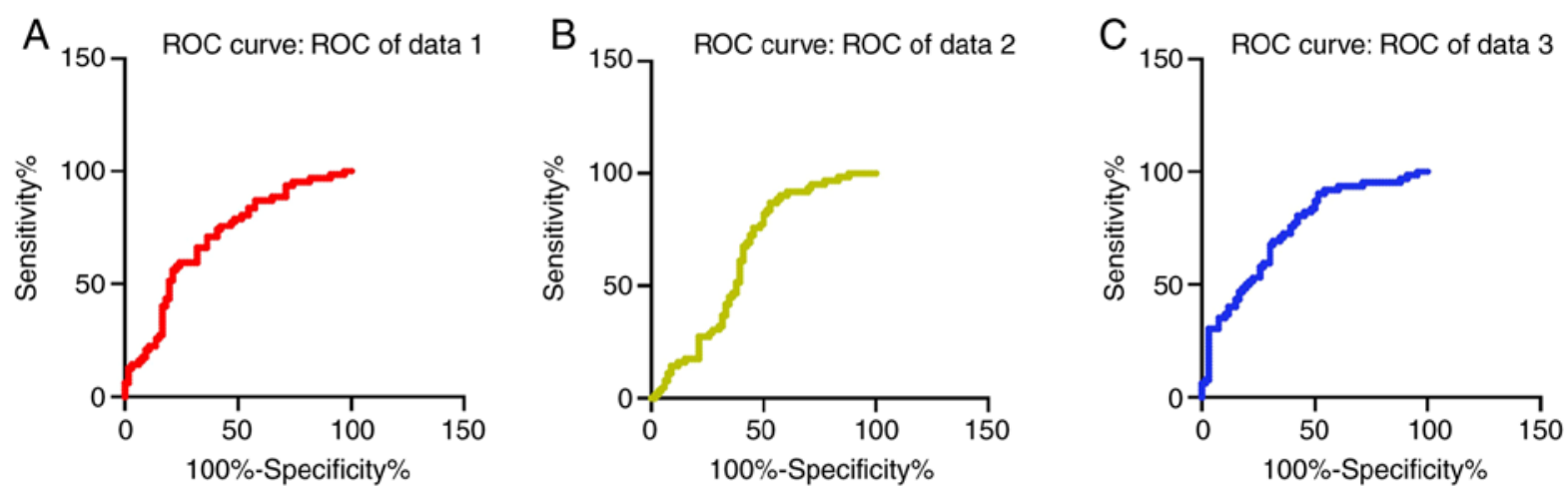

Figure 1. Analysis of serum CTRP9 level in the diagnostic efficacy of restenosis after cerebrovascular stent implantation. (A) ROC curve analysis showed that the sensitivity of serum CTRP9 level in the diagnosis of restenosis after cerebrovascular stent implantation was 59.68\%, the specificity was $75.76 \%$, AUC was 0.705 and cut off was 180.6. (B) ROC curve analysis showed that the sensitivity of serum STIM1 level in diagnosis of restenosis after cerebrovascular stent implantation was $87.10 \%$, the specificity was $46.97 \%$, AUC was 0.637 and cut off was 12.24 . (C) The pre-1 fitting of probability value in Logistics regression model combined ROC curve results showed that the sensitivity of serum CTRP9 and STIM1 levels in the combined diagnosis of restenosis after cerebrovascular stent implantation was $90.32 \%$, the specificity was $48.48 \%$, AUC was 0.747 and cut off was 0.484 .

significantly lower than that in group B. Serum STIM1 level in group A was significantly higher than that in group $\mathrm{B}$. The differences were statistically significant (both $\mathrm{P}<0.001$ ). More details are shown in Table II.

Comparison of NO, TNF- $\alpha, I L-6$ levels of patients in the two groups. Serum NO level in group A was significantly lower than that in group B. Serum TNF- $\alpha$ and IL-6 levels in group A were significantly higher than those in group B. The differences were statistically significant (all $\mathrm{P}<0.001$ ). More details are shown in Table III.

Comparison of serum CTRP9, STIM1 levels and combined diagnostic efficacy by ROC curve analysis. The sensitivity, specificity, AUC and cut-off of serum CTRP9 level in the diagnosis of restenosis after cerebrovascular stent implantation were 59.68, 75.76, 0.705 and $180.6 \%$, respectively. The sensitivity, specificity, AUC and cut-off of serum STIM1 level in diagnosis of restenosis after cerebrovascular stent implantation were $87.10,46.97,0.637$ and $12.24 \%$, respectively. The sensitivity, specificity, AUC and cut-off of the combination of serum CTRP9 and STIM1 levels in the combined diagnosis of restenosis after cerebrovascular stent implantation were 90.32, 48.48, 0.747 and $0.484 \%$, respectively (Fig. 1).

Analysis of the correlation between serum CTRP9, STIM1 levels and NO,TNF- $\alpha, I L-6$ levels. After cerebrovascular stent implantation, the level of CTRP9 was positively correlated with NO ( $\mathrm{r}=0.711, \mathrm{P}<0.001)$, and negatively correlated with TNF- $\alpha(r=-0.761, \mathrm{P}<0.001)$ and IL-6 $(r=-0.751, \mathrm{P}<0.001)$. The level of STIM1 was negatively correlated with NO ( $\mathrm{r}=-0.761$, $\mathrm{P}<0.001)$, and positively correlated with TNF- $\alpha(\mathrm{r}=0.776$, $\mathrm{P}<0.001)$ and IL-6 (r=0.709, $\mathrm{P}<0.001)$ (Fig. 2).

\section{Discussion}

Many studies have shown that the incidence of postoperative in-stent restenosis is higher than that of non-operative surgery $(13,14)$. However, the mechanism of restenosis after cerebrovascular stent implantation remain unclear. Most 

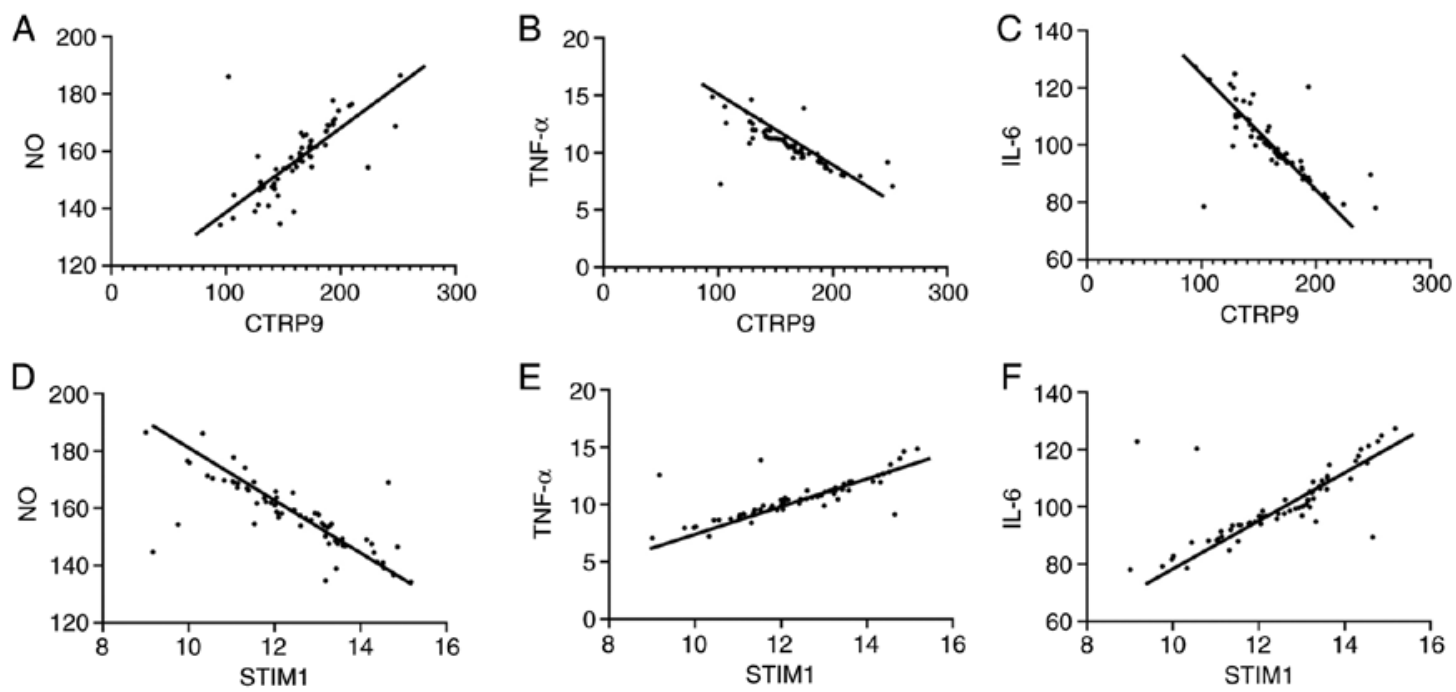

Figure 2. Analysis of the correlation between serum CTRP9, STIM1 levels and NO, TNF- $\alpha$, IL-6 levels after cerebrovascular stent implantation. (A) Analysis of the correlation between serum CTRP9 levels and NO levels after cerebrovascular stent implantation. Pearson's analysis showed that the expression of CTRP9 and NO was positively correlated ( $r=0.711, \mathrm{P}<0.001)$. (B) Analysis of the correlation between serum CTRP9 levels and TNF- $\alpha$ levels after cerebrovascular stent implantation. Pearson analysis showed that the expression of CTRP9 and TNF- $\alpha$ was negatively correlated $(r=-0.761, \mathrm{P}<0.001)$. (C) Analysis of the correlation between serum CTRP9 levels and IL-6 levels after cerebrovascular stent implantation. Pearson's analysis showed that the expression of CTRP9 and IL-6 was negatively correlated ( $\mathrm{r}=-0.751, \mathrm{P}<0.001)$. (D) Analysis of the correlation between serum STIM1 levels and NO levels after cerebrovascular stent implantation. Pearson's analysis showed that the expression of STIM1 and NO was negatively correlated $(\mathrm{r}=-0.761, \mathrm{P}<0.001)$. (E) Analysis of the correlation between serum STIM1 levels and TNF- $\alpha$ levels after cerebrovascular stent implantation. Pearson's analysis showed that the expression of STIM1 and TNF- $\alpha$ was positively correlated $(\mathrm{r}=0.776, \mathrm{P}<0.001)$. (F) Analysis of the correlation between serum STIM1 levels and IL-6 levels after cerebrovascular stent implantation. Pearson's analysis showed that the expression of STIM1 and IL- 6 was positively correlated $(r=0.709, P<0.001)$. NO, nitric oxide; TNF- $\alpha$, tumor necrosis factor $\alpha$; IL-6; interleukin-6.

scholars agree that it is related to vascular remodeling, intima abnormal growth, polymer absorption hindering inflammatory response and postoperative vascular elastic retraction and other factors $(15,16)$. It is of practical significance to find out the cause of restenosis after cerebrovascular stent implantation. It has been reported (17) that STIM1 is a sensory receptor for calcium channel of calcium store controllability in vivo. The biological effects of cells are affected by controlling intracellular calcium concentration to regulate vasoconstriction. In vitro experiments have shown that CTRP9 can inhibit the proliferation of smooth muscle cells and angiogenesis and the formation of vascular new intima through Cyclic AMP (cAMP)-independent mechanism after injury of vascular (18). The increase of nitric oxide (NO) production promotes vasodilation and greatly improves endothelial cell function (19). These effects may be related to STIM1, CTRP9 and restenosis after cerebrovascular stenting. Therefore, this study investigated the effects of serum CTRP9 and STIM1 on restenosis after cerebrovascular stent implantation, and explored their relationship with vasoactive substances and inflammatory cytokines.

Based on the results of this study, serum CTRP9 level in group A was significantly lower than that in group B, and serum STIM1 level in group A was significantly higher than that in group $\mathrm{B}$ (all $\mathrm{P}<0.001)$, suggesting that serum CTRP9 low expression and STIM1 over-expression after cerebrovascular stent implantation may increase the risk of in-stent restenosis. When STIM1 expression is released, it inhibits the biological and homing functions of endothelial progenitor cells, directly damaging the repair of local vascular injury after stent implantation (20). According to a previous study (21), the increase of free fatty acids during tissue ischemia/reperfusion injury promotes the oxidative stress and increase of expression of CTRP9, and improves the metabolism function of the body to protect damaged myocardial cells, which is consistent with our results. The levels of serum NO, TNF- $\alpha$ and IL- 6 in group A were significantly higher than those in group $\mathrm{B}$ (all $\mathrm{P}<0.001)$. Pearson results showed that serum CTRP9 level was positively correlated with NO and negatively correlated with TNF- $\alpha$ and IL-6. STIM1 was positively correlated with TNF- $\alpha$ and IL-6 and negatively correlated with NO (all $\mathrm{P}<0.001$ ). The sustainable or controlled release of NO has diastolic effect on the endothelium and can repair and accelerate the vascular regeneration (22). TNF- $\alpha$ can regulate the expression of vasomotion substances and cause vasoconstriction after the decrease of the levels of vasorelaxation factors $(23,24)$. It has been reported that IL- 6 is involved in tissue fibrosis and induces the release of a large amount of inflammatory mediators under the effect of vascular endothelial tissue injury (25). In summary, serum TNF- $\alpha$ and IL- 6 levels increase with vascular stenosis, while NO expression decreases. Combined with the results of CTRP9 and STIM1, the results of correlation between serum CTRP9, STIM1 levels and NO, TNF- $\alpha$ and IL- 6 were inferred and confirmed, which further confirmed the predictive value of serum CTRP9 and STIM1 levels on restenosis after cerebrovascular stent implantation. The sensitivity of serum CTRP9 level in the diagnosis of restenosis after cerebrovascular stent implantation was $59.68 \%$, and the specificity was $75.76 \%$. The sensitivity of serum STIM1 level in diagnosis of restenosis after cerebrovascular stent implantation was $87.10 \%$ and the specificity was $46.97 \%$. The sensitivity of serum CTRP9 and STIM1 levels in the combined diagnosis of restenosis after 
cerebrovascular stent implantation was $90.32 \%$ and the specificity was $48.48 \%$. It indicated that both CTRP9 and STIM1 may be involved in the disease process of pathophysiological mechanism of restenosis after cerebrovascular stent implantation. The regulation and protection of CTRP9 and STIM1 on cerebrovascular function result in abnormal changes of intravascular components after reversal. There is substantial heterogeneity in the influence of signaling pathways and action sites, and the emphasis indicates that metabolic regulation is also different. Synergistic combined detection may improve the diagnostic efficacy of cardiovascular and cerebrovascular diseases.

Based on previous studies and the results of this study, it can be learned that the decreased level of CTRP9 and increased level of STIM1 after cerebrovascular stent implantation are closely related to vascular formation, repair and re-endothelialization. However, there are some limitations in this study. The specific differences between the combined diagnosis of CTRP9 and STIM1 and the separate diagnosis are not discussed in detail. We did not rule out the interference of other factors on these indexes, and failed to use the two factors as early predictors of restenosis after cerebral vascular stenting. These are the research topics of the future study. However, the results of this study have preliminarily shown that CTRP9 and STIM1 may play a role in the early prevention of restenosis after cerebrovascular stent implantation, providing a new theoretical direction for the diagnosis and prognostic evaluation of restenosis after cerebrovascular stent implantation.

\section{Acknowledgements}

Not applicable.

\section{Funding}

No funding was received.

\section{Availability of data and materials}

The datasets used and/or analyzed during the present study are available from the corresponding author on reasonable request.

\section{Authors' contributions}

JP, XC and LZ conceived and designed the study. JP, GW, KX, $\mathrm{JH}$ and $\mathrm{LZ}$ were responsible for the acquisition, analysis and interpretation of the data. JP drafted the manuscript. XC and $\mathrm{LZ}$ revised the manuscript critically for important intellectual content. All authors read and approved the final manuscript.

\section{Ethics approval and consent to participate}

The study was approved by the Ethics Committee of Yantaishan Hospital (Yantai, China). Signed informed consents were obtained from the patients and/or guardians.

\section{Patient consent for publication}

Not applicable.

\section{Competing interests}

The authors declare that they have no competing interests.

\section{References}

1. Losito A, Pittavini L, Ferri C and De Angelis L: Kidney function and mortality in different cardiovascular diseases: Relationship with age, sex, diabetes and hypertension. J Nephrol 24: 322-328, 2011.

2. Sairanen T, Strbian D, Ruuskanen R, Silvennoinen H, Salonen O and Lindsberg PJ: Symptomatic intracranial haemorrhage after thrombolysis with adjuvant anticoagulation in basilar artery occlusion. Eur J Neurol 22: 493-499, 2015.

3. Mintz GS: Intravascular ultrasound and outcomes after drugeluting stent implantation. Coron Artery Dis 28: 346-352, 2017.

4. Guan CL, Shi GX, Li Q, Luo YM and Zeng YC: Prevention of complications following cerebrovascular stent implantation. J Clin Rehab Tissue Eng Res 2 (Suppl): S52, 2010.

5. Dinesch V, Dinesch M and Buruian M: Drug-eluting stent in-stent restenosis and re-restenosis: A hard time story. J Saudi Heart Assoc 29: 73-74, 2017.

6. Ouchi N, Parker JL, Lugus JJ and Walsh K: Adipokines in inflammation and metabolic disease. Nat Rev Immunol 11: 85-97, 2011.

7. Lago F, Dieguez C, Gómez-Reino J and Gualillo O: Adipokines as emerging mediators of immune response and inflammation. Nat Clin Pract Rheumatol 3: 716-724, 2007.

8. Smith CC and Yellon DM: Adipocytokines, cardiovascular pathophysiology and myocardial protection. Pharmacol Ther 129: 206-219, 2011.

9. Schäffler A and Buechler C: CTRP family: Linking immunity to metabolism. Trends Endocrinol Metab 23: 194-204, 2012.

10. Wong GW, Krawczyk SA, Kitidis-Mitrokostas C, Ge G, Spooner E, Hug C, Gimeno R and Lodish HF: Identification and characterization of CTRP9, a novel secreted glycoprotein, from adipose tissue that reduces serum glucose in mice and forms heterotrimers with adiponectin. FASEB J 23: 241-258, 2009.

11. Liou J, Fivaz M, Inoue T and Meyer T: Live-cell imaging reveals sequential oligomerization and local plasma membrane targeting of stromal interaction molecule 1 after $\mathrm{Ca}^{2+}$ store depletion. Proc Natl Acad Sci USA 104: 9301-9306, 2007.

12. Estrada IA, Donthamsetty R, Debski P, Zhou MH, Zhang SL, Yuan JX, Han W and Makino A: STIM1 restores coronary endothelial function in type 1 diabetic mice. Circ Res 111: 1166-1175, 2012.

13. Edgell RC, Zaidat OO, Gupta R, Abou-Chebl A, Linfante I, Xavier A, Nogueira R, Alshekhlee A, Kalia J, Etezadi V, et al: Multicenter study of safety in stenting for symptomatic vertebral artery origin stenosis: Results from the Society of Vascular and Interventional Neurology Research Consortium. J Neuroimaging 23: 170-174, 2013.

14. Albuquerque FC, Fiorella D, Han P, Spetzler RF and Mc Dougall CG: A reappraisal of angioplasty and stenting for the treatment of vertebral origin stenosis. Neurosurgery 53: 607-616, 2003.

15. Kang SJ, Mintz GS, Park DW, Lee SW, Kim YH, Lee CW, Han KH, Kim JJ, Park SW and Park SJ: Mechanisms of in-stent restenosis after drug-eluting stent implantation: Intravascular ultrasound analysis. Circ Cardiovasc Interv 4: 9-14, 2011.

16. Virmani R, Liistro F, Stankovic G, Di Mario C, Montorfano M, Farb A, Kolodgie FD and Colombo A: Mechanism of late in-stent restenosis after implantation of a paclitaxel derivate-eluting polymer stent system in humans. Circulation 106: 2649-2651, 2002.

17. Peel SE, Liu B and Hall IP: A key role for STIM1 in store operated calcium channel activation in airway smooth muscle. Respir Res 7: 119, 2006.

18. Zheng Q, Yuan Y, Yi W, Lau WB, Wang Y, Wang X, Sun Y, Lopez BL, Christopher TA, Peterson JM, et al: Clq/TNF-related proteins, a family of novel adipokines, induce vascular relaxation through the adiponectin receptor-1/AMPK/e NOS/nitric oxide signaling pathway. Arterioscler Thromb Vasc Biol 31: 2616-2623, 2011.

19. Takeuchi S, Wada K, Uozumi Y, Otani N, Osada H, Nagatani K and Mori K: Adiponectin receptor 1 expression is associated with carotid plaque stability. Neurol India 61: 249-253, 2013.

20. Jing XD, Wei XM, Deng SB, Du JL, Liu YJ and She Q: The relationship between the high-density lipoprotein (HDL)-associated sphingosine-1-phosphate (S1P) and coronary in-stent restenosis. Clin Chim Acta 15: 248-252, 2015. 
21. Kambara T, Ohashi K, Shibata R, Ogura Y, Maruyama S, Enomoto T, Uemura Y, Shimizu Y, Yuasa D, Matsuo K, et al: CTRP9 protein protects against myocardial injury following ischemia-reperfusion through AMP-activated protein kinase (AMPK)-dependent mechanism. J Biol Chem 287: 18965-18973, 2012.

22. Elnaggar MA, Seo SH, Gobaa S, Lim KS, Bae IH, Jeong MH, Han DK, Joung YK: Nitric oxide releasing coronary stent: A new approach using layer-by-layer coating and liposomal encapsulation. Small 12: 6012-6023, 2016.

23. Zhang H, Park Y, Wu J, Chen XP, Lee S, Yang J, Dellsperger KC and Zhang C: Role of TNF-alpha in vascular dysfunction. Clin Sci (Lond) 116: 219-230, 2009.
24. Picchi A, Gao X, Belmadani S, Potter BJ, Focardi M, Chilian WM and Zhang C: Tumor necrosis factor-alpha induces endothelial dysfunction in Lepr (dp) mice. Circulation 115: 245-254, 2007.

25. Booth AJ, Csencsits-Smith K, Wood SC, Lu G, Lipson KE and Bishop DK: Connective tissue growth factor promotes fibrosis downstream of TGFbeta and IL-6 in chronic cardiac allograft rejection. Am J Transplant 10: 220-230, 2010. 